\title{
Mechanisms of improving coastal saline-alkali soil by periphyton
}

\author{
Ye Zhu ${ }^{1}$, Tianyun Shao ${ }^{1}$, Yujie Zhou ${ }^{1}$, Xiumei Gao $^{1}$, Xiaohua Long ${ }^{1}$, and Zed Rengel ${ }^{2}$ \\ ${ }^{1}$ Nanjing Agricultural University \\ ${ }^{2}$ The University of Western Australia
}

January 20, 2021

\begin{abstract}
Periphyton plays an indispensable role in coastal saline-alkali land, but its function is poorly understood. Soil physical and chemical properties ( $\mathrm{pH}$ value, salinity, soil organic matter), enzyme activity and microbial diversity (based on 16s rDNA, ITS and functional genes) were measured in periphyton formed on rice-growing coastal saline-alkali soil modified by a new type of soil conditioner. The results showed that the content of organic matter and catalase activity in periphyton were significantly higher than in the unplanted control soil. Soil pH and salinity were decreased in periphyton compared to the unplanted control soil. Based on the relative abundance, bacterial genera Desulfomicrobium, Rhodobacter, cyanobacterium_scsio_T-2, Gemmatimonas, and Salinarimonas as well as fungal genus Fusarium were more abundant in periphyton than the unplanted control soil. In terms of functional genes, the cbbM and cbbL sequencing showed higher abundance of Hydrogenophaga, Rhodovulum, Magnetospira, Leptothrix, and Thiohalorhabdus, whereas the nifH sequencing indicated higher abundance of Cyanobacteria in the periphyton compared to the unplanted soil. The relative abundance and community structure of soil microorganisms were improved by periphyton, thus reducing soil salinity and $\mathrm{pH}$, increasing soil organic matter and enzyme activity. This indicated that the periphyton can improve the conditions and offer a suitable environment for plant growth in coastal saline-alkali soil.
\end{abstract}

\section{Hosted file}

Periphyton LDD.pdf available at https://authorea.com/users/390765/articles/504989-mechanismsof-improving-coastal-saline-alkali-soil-by-periphyton

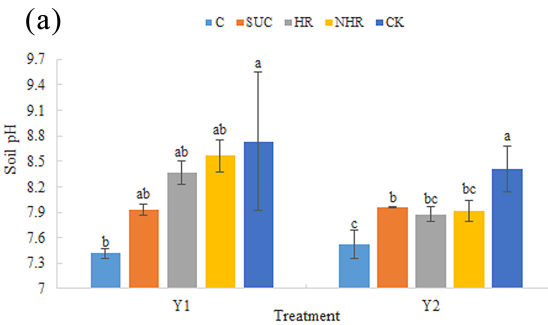

(c)

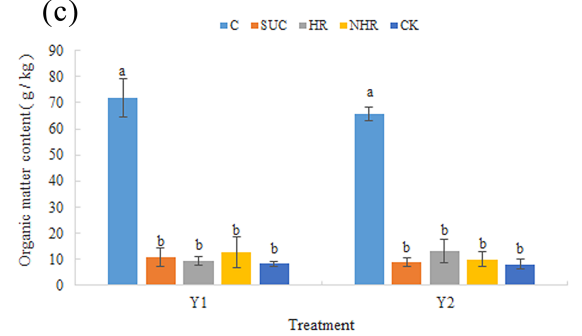

(b) $\quad$ "C $=\mathrm{BSUC} " \mathrm{HR}=\mathrm{NHR}$ " $\mathrm{CKK}$

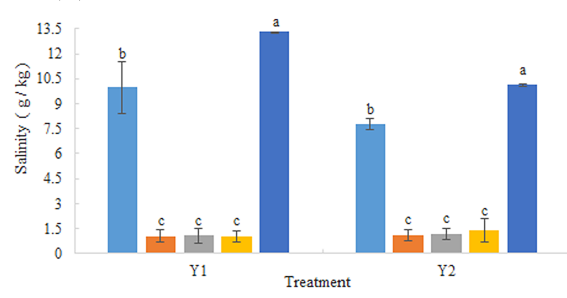

(d)

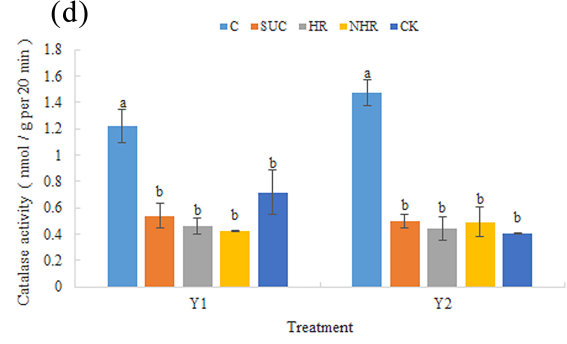


16s rRNA sequencing

(a)

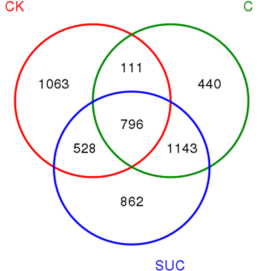

(c)

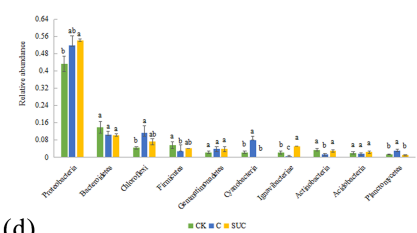

(d)

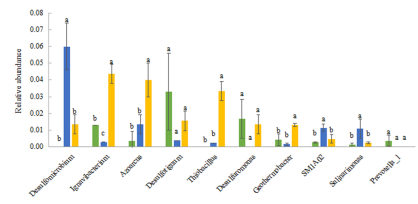

(b)

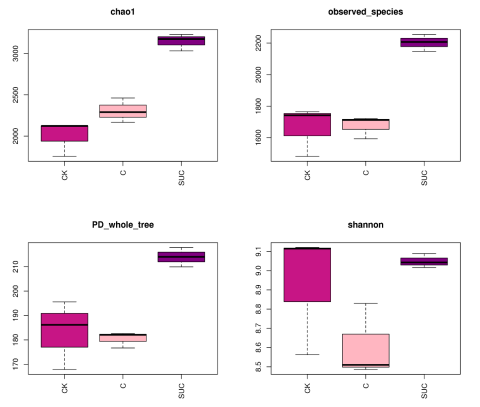

(e)

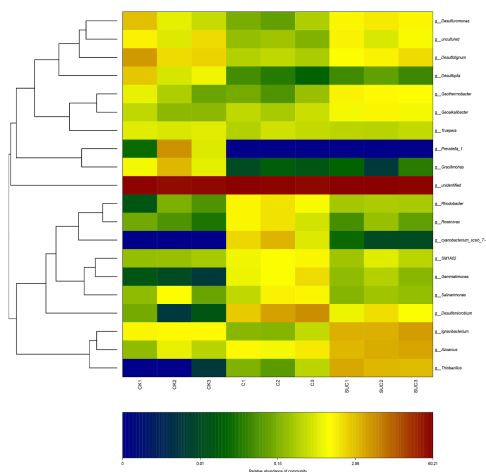

(g)

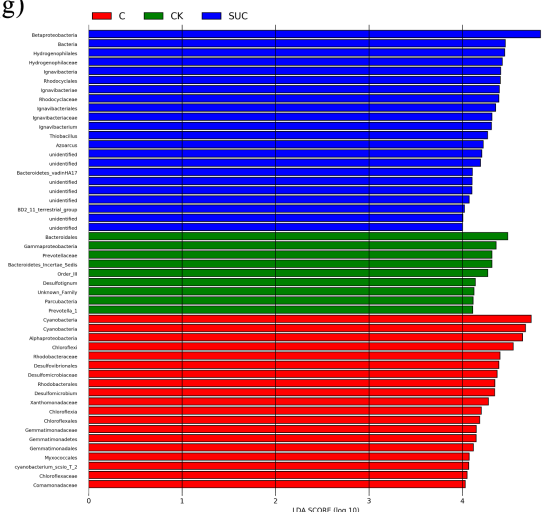




\section{ITS sequencing}

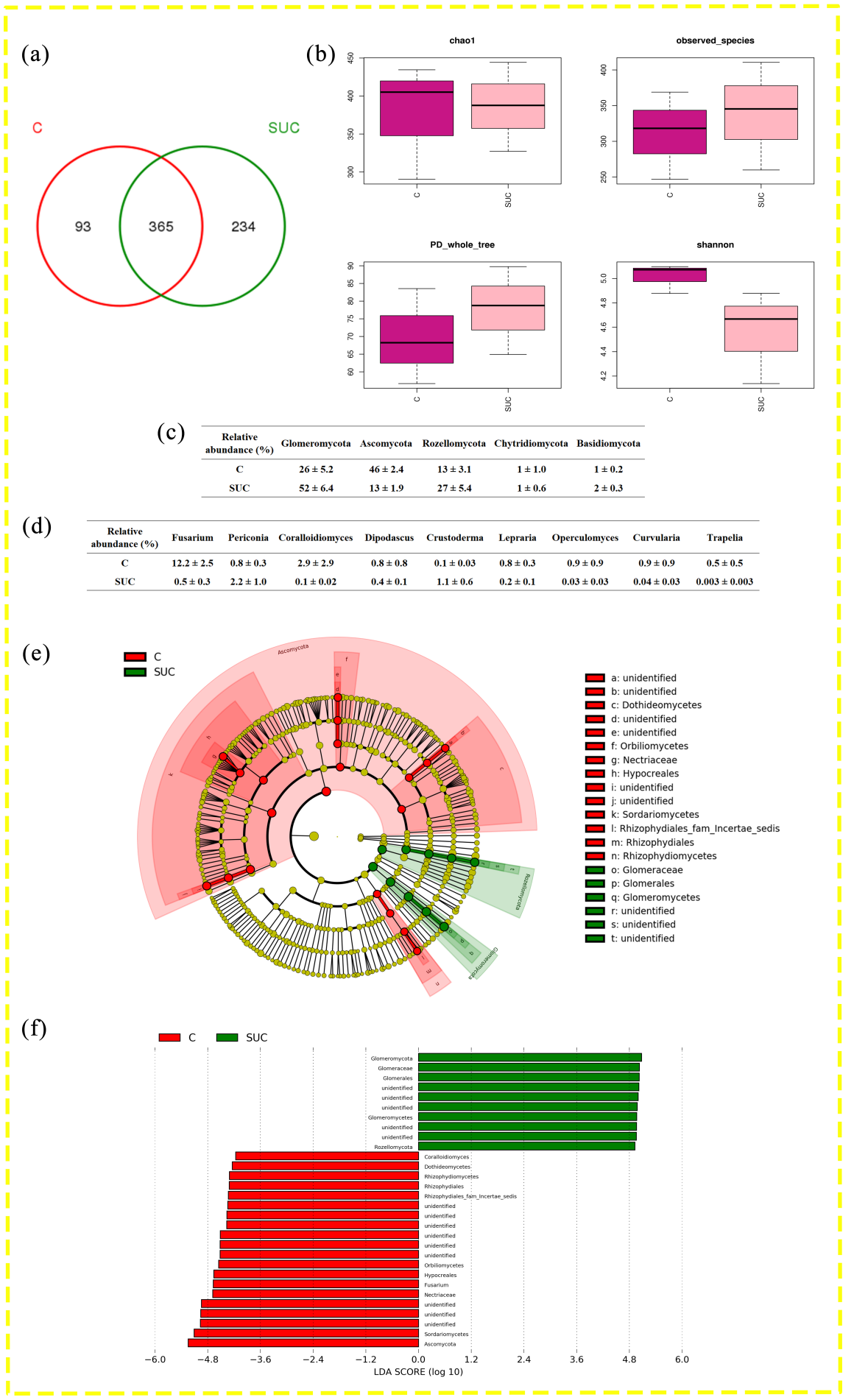




\section{$c b b L$ sequencing}

(a)

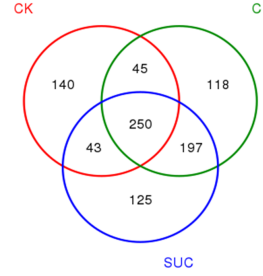

(c)

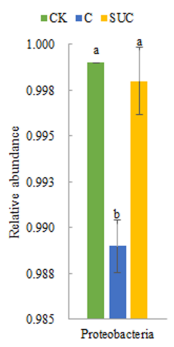

(b)
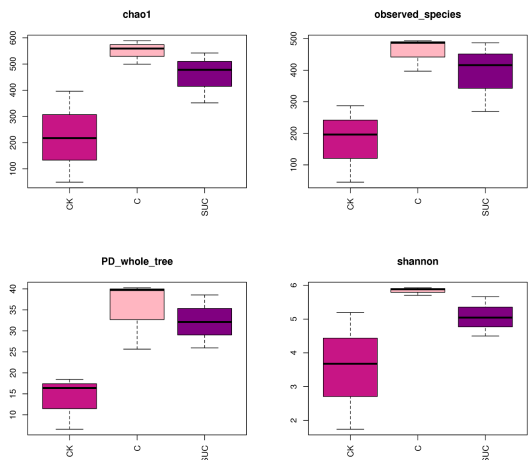

(d)

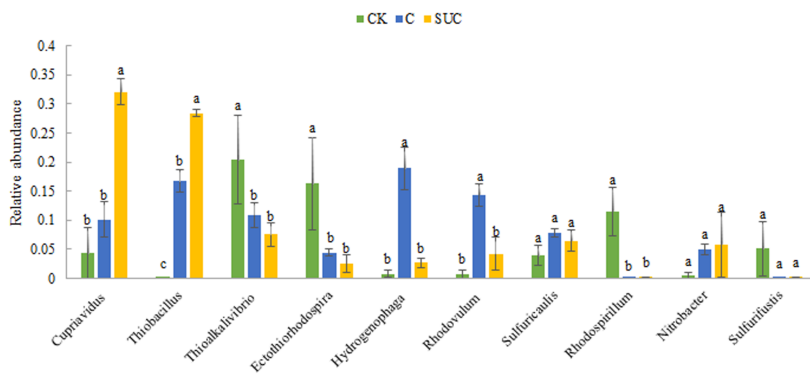

(e) 吕 suc

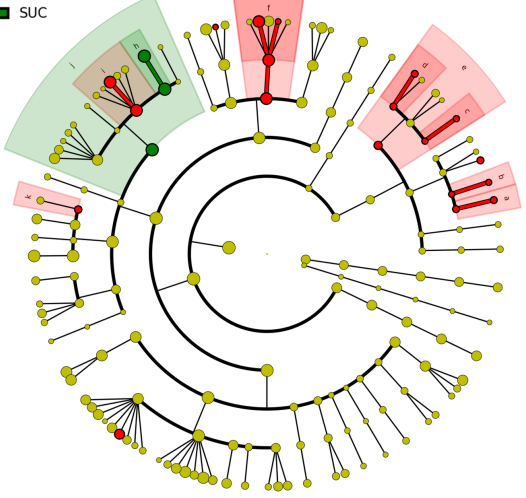

a: Coleofasciculacea

b: Cyanothecaceae c: Prochlorotrichaceae d: Synechococcaceae

f: Rhodobacteracea

g: Rhodobacterales

i: Comamonadacea

j: Burkholderiales

(f)
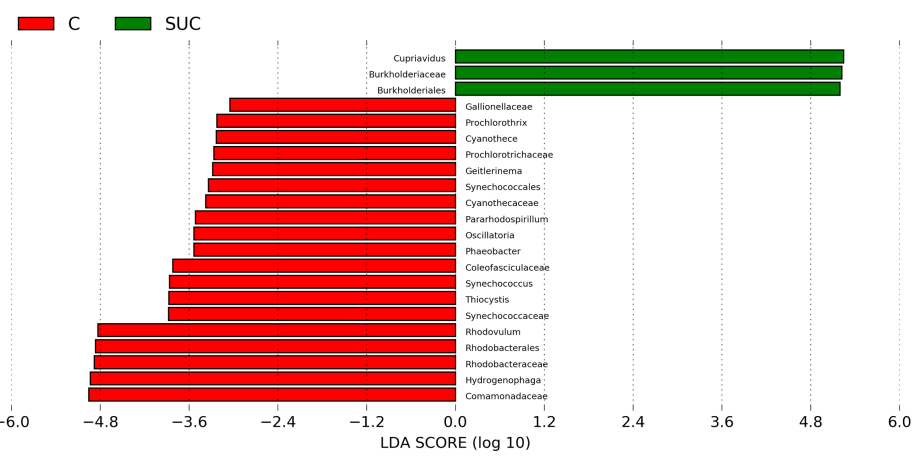
$c b b M$ sequencing

(a)

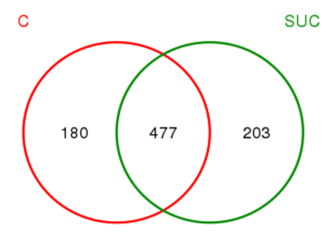

(b)
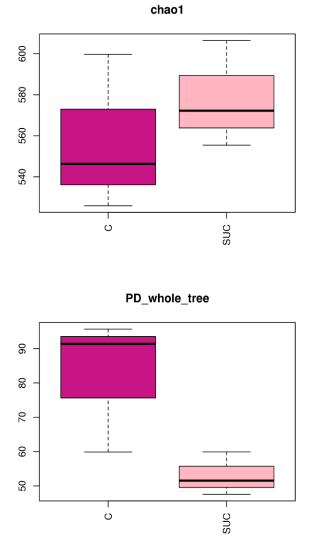

(c)
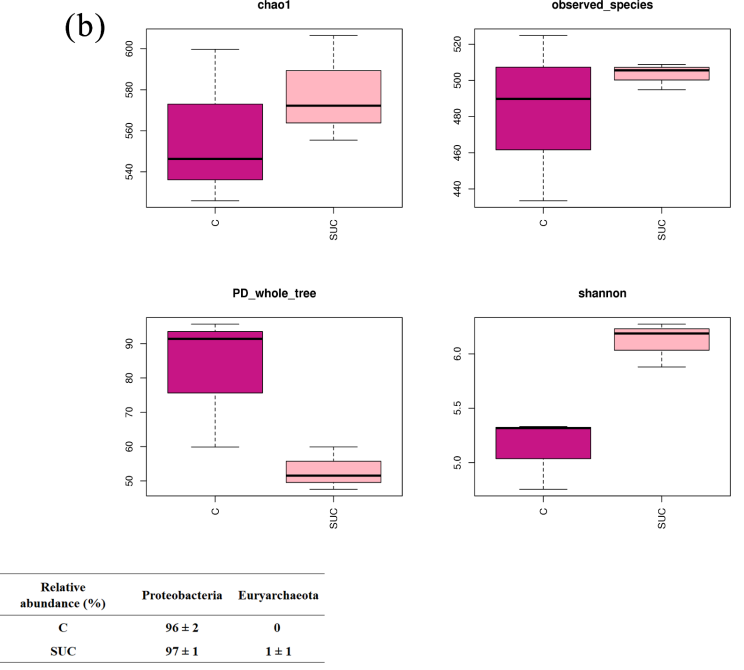

(d)

\begin{tabular}{|c|c|c|c|c|c|c|c|c|c|c|}
\hline $\begin{array}{c}\text { Relative } \\
\text { abundance (\%) }\end{array}$ & Magnetospira & Thiobacillus & Sulfuritalea & Leptothrix & Acidithiobacillus & Acidihalobacter & Thiohalorhabdus & Halothiobacillus & Thioalkalicoccus & Thiohalomonas \\
\hline c & $56.2 \pm 4.5$ & $7.6 \pm 0.9$ & $1.8 \pm 0.9$ & 3.3 & 1.2 & 2.5 & $4.0 \pm 0.5$ & $0.9 \pm 0.1$ & $0.8 \pm 0.1$ & $1.2 \pm 0.2$ \\
\hline suc & $.4 \pm 8.8$ & $2.9 \pm 2.8$ & $10.7 \pm 6.7$ & $4.9 \pm 1.3$ & $7.6 \pm 2.9$ & $4.8 \pm 0.5$ & $2.9 \pm 2.0$ & $5.5 \pm 3.1$ & $2.1 \pm 0.8$ & $1.3 \pm 1.5$ \\
\hline
\end{tabular}

(e)
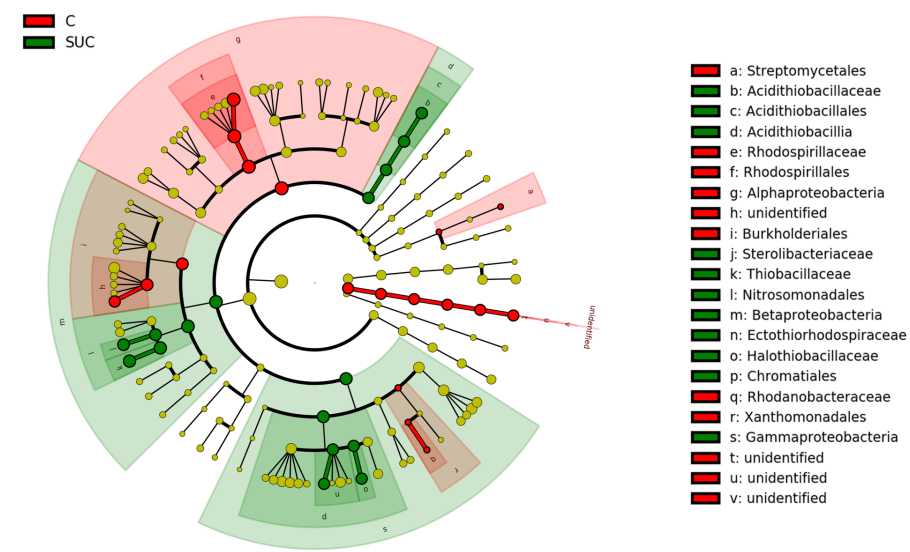

(f)

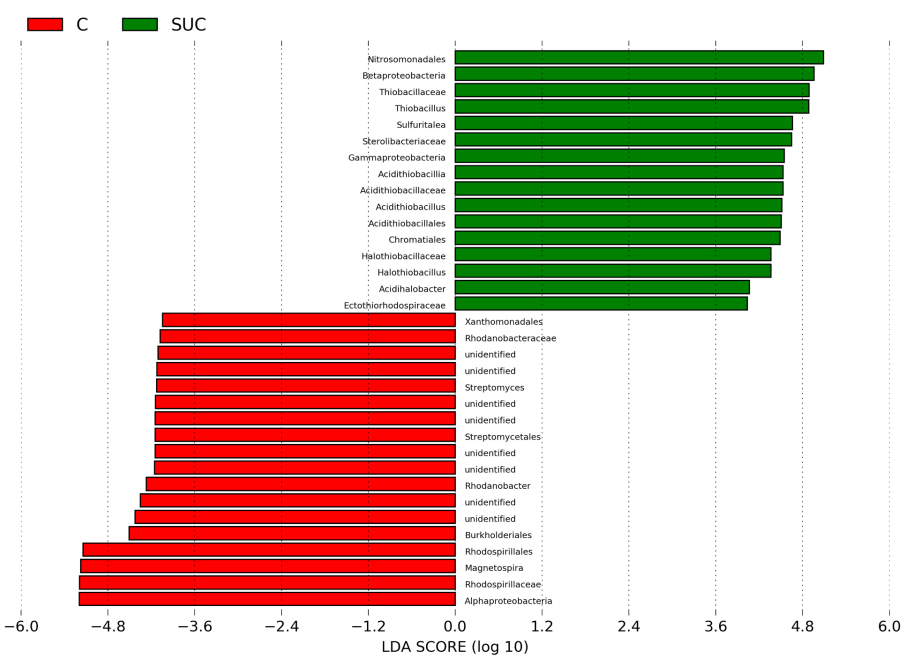


nifH sequencing

(a)
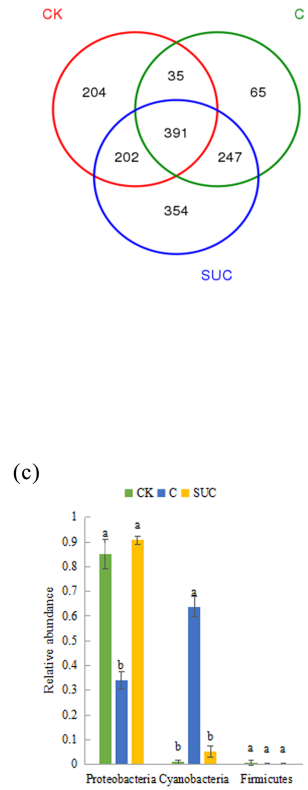

(e)

$\mathrm{g}_{\mathrm{g}}^{\mathrm{c}} \mathrm{suc}$
$\mathrm{suc}^{\mathrm{n}}$
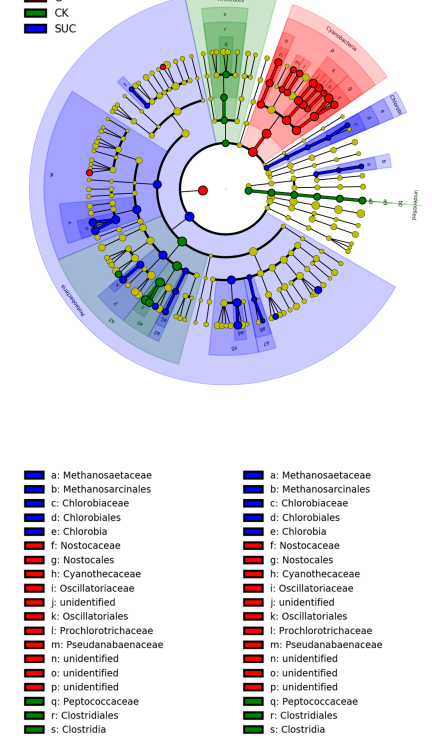

(b)

(d)
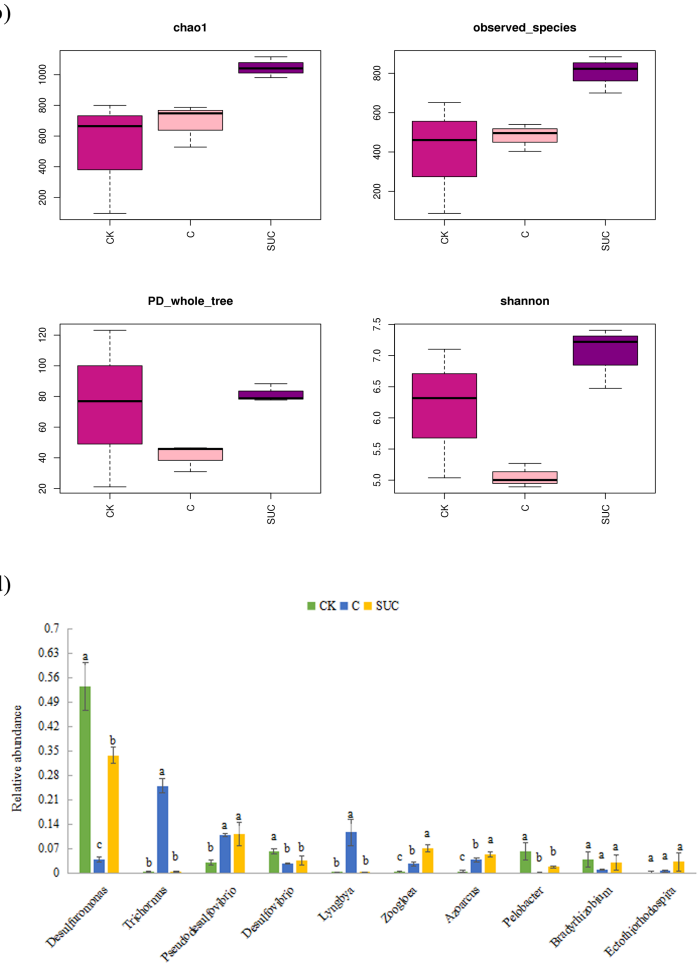

(f) $\boldsymbol{D}_{\mathrm{c}} \boldsymbol{\square}_{\mathrm{ck}} \boldsymbol{\nabla}_{\mathrm{suc}}$

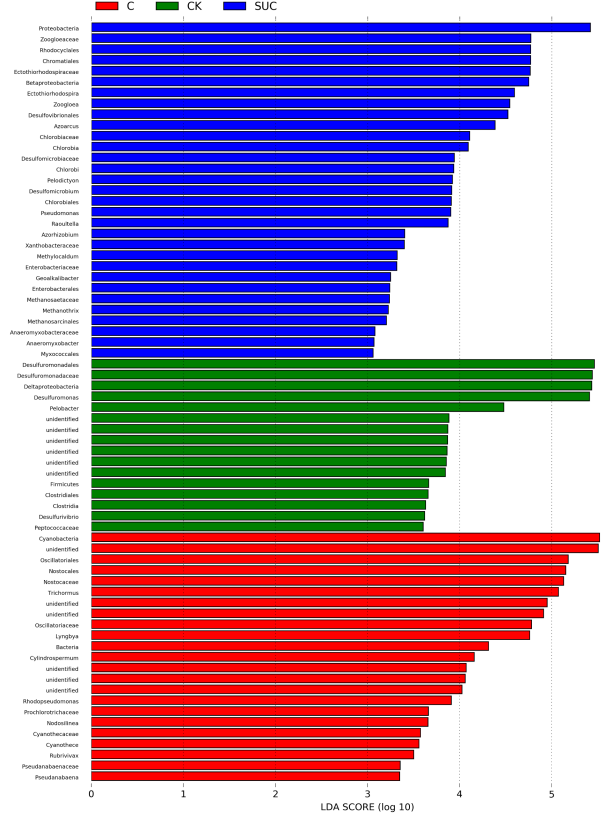




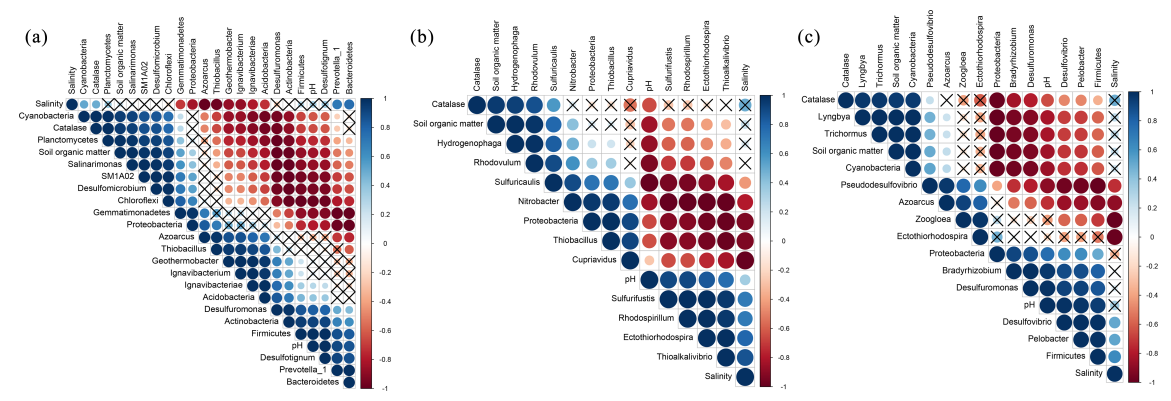

